\title{
Supermodular ordering of Poisson arrays
}

\author{
Bünyamin Kızıldemir* Nicolas Privault ${ }^{\dagger}$ \\ Division of Mathematical Sciences \\ School of Physical and Mathematical Sciences \\ Nanyang Technological University \\ 637371 Singapore
}

January 8, 2015

\begin{abstract}
We derive necessary and sufficient conditions for the supermodular ordering of certain triangular arrays of Poisson random variables, based on the componentwise ordering of their covariance matrices. Applications are proposed for markets driven by jump-diffusion processes, using sums of Gaussian and Poisson random vectors. Our results rely on a new triangular structure for the representation of Poisson random vectors using their Lévy-Khintchine representation.
\end{abstract}

Key words: Stochastic ordering; supermodular functions; infinitely divisible random vectors; Poisson distribution; jump-diffusion models.

Mathematics Subject Classification: 60E15; 62H20; 60E07, 91B28.

\section{Introduction}

Stochastic ordering of random vectors is used in finance and economics as a risk management tool that yields finer information than mean-variance analysis. A $d$ dimensional random vector $X=\left(X_{1}, \ldots, X_{d}\right)$ is said to be dominated by another random vector $Y=\left(Y_{1}, \ldots, Y_{d}\right)$ if

$$
E[\Phi(X)] \leqslant E[\Phi(Y)],
$$

for all sufficiently integrable $\Phi: \mathbb{R}^{d} \rightarrow \mathbb{R}$ within a certain class of functions that determines the ordering.

\footnotetext{
*bunyamin001@e.ntu.edu.sg
}

${ }^{\dagger}$ nprivault@ntu.edu.sg 
One writes $X \leqslant_{\mathrm{sm}} Y$ when (1.1) holds for all supermodular functions, i.e. for every function $\Phi: \mathbb{R}^{d} \longrightarrow \mathbb{R}$ such that

$$
\Phi(x)+\Phi(y) \leqslant \Phi(x \wedge y)+\Phi(x \vee y), \quad x, y \in \mathbb{R}^{d}
$$

where the maximum $\vee$ and the minimum $\wedge$ defined with respect to the componentwise order, cf. [6] and references therein for a review with economic interpretations.

It is well-known (cf. e.g. Theorem 3.9.5 of [10]) that necessary conditions for $X \leqslant{ }_{\mathrm{sm}} Y$ are:

(i) $X_{i}$ and $Y_{i}$ have same distribution for all $i=1, \ldots, d$, and

(ii) for all $1 \leqslant i<j \leqslant d$ we have

$$
\operatorname{Cov}\left(X_{i}, X_{j}\right) \leqslant \operatorname{Cov}\left(Y_{i}, Y_{j}\right)
$$

where $(i)$ above follows from the fact that any function of a single variable is supermodular, and $(i i)$ follows by application of (1.1) to the supermodular function $\Phi_{i, j}\left(x_{1}, \ldots, x_{d}\right):=\left(x_{i}-E\left[X_{i}\right]\right)\left(x_{j}-E\left[X_{j}\right]\right), 1 \leqslant i<j \leqslant d$.

Supermodular ordering of Gaussian random vectors has been completely characterized in [9] Theorem 4.2, cf. also Theorem 3.13.5 of [10], by showing that $(i)$ and $(i i)$ above are also sufficient conditions when $X$ and $Y$ are Gaussian, cf. also [8] for other orderings (stochastic, convex, convex increasing, supermodular) of Gaussian random vectors.

In this paper we consider the supermodular ordering of vectors of Poisson random variables, see [12], [5] for early references on the multivariate Poisson distribution. As noted in Section 2, any $d$-dimensional Poisson random vector is based on $2^{d}-1$ parameters, therefore $(i)$ and $(i i)$, which are based on $d(d+1) / 2$ conditions, cannot characterize their distribution ordering except if $d=2$.

For this reason, in Section 3 we consider a particular dependence structure of Poisson arrays depending on $d(d+1) / 2$ parameters based on a natural decomposition of 
their Lévy measure on the vertices of the $d$-dimensional unit hypercube. We show in Theorem 4.2 that in this case, conditions $(i)$ and (ii) become necessary and sufficient for the supermodular ordering of $X$ and $Y$ as in the Gaussian setting. When $d=2$, no restriction has to be imposed on 2-dimensional Poisson random vectors $X$ and $Y$. Triangular Poisson structures of a different type have been considered in [11] for the simulation of Poisson random vectors.

Our proof relies on the characterization of the supermodular ordering of $d$-dimensional infinitely divisible random vectors $X$ and $Y$ by their Lévy measures, cf. [2], based on the covariance identities of [4]. Extensions of such identities have already been applied to other multidimensional stochastic (including convex) orderings in e.g. [3] based on stochastic calculus and in [1] using forward-backward stochastic calculus.

We also derive sufficient conditions for the supermodular ordering of sums of Gaussian and Poisson random vectors, with application to a jump-diffusion asset price model, cf. Theorem 4.4. Indeed, the supermodular ordering of random asset vectors implies the stop-loss ordering of their associated portfolios, cf. Theorem 3.1 in [7] or Theorem 8.3 .3 in $[10]$.

We proceed as follows. In Section 2 we recall the construction of Poisson random vectors, and in Section 3 we specialize this construction to a certain dependence setting based on Poisson arrays. Finally in Section 4 we prove our main characterization of supermodular ordering for such vectors, including extensions to the increasing supermodular order, cf. Theorem 4.2 and Proposition 4.3. In addition we provide a sufficient condition for the supermodular ordering of sums of Gaussian and Poisson random vectors in Theorem 4.4, with application to an exponential jump-diffusion asset price model. We also include a remark on the related convex ordering problem for such Poisson arrays in Proposition 4.5. 


\section{Poisson random vectors}

Consider $X=\left(X_{i}\right)_{1 \leqslant i \leqslant d}$ a $d$-dimensional infinitely divisible Poisson random vector with Lévy measure $\mu$ on $\mathbb{R}^{d}$, which satisfies

$$
E\left[e^{i\langle\bar{t}, X\rangle}\right]=\exp \left(\int_{\mathbb{R}^{d}}\left(e^{i\langle\bar{t}, x\rangle}-1\right) \mu(d x)\right),
$$

where $\bar{t}=\left(t_{1}, \ldots, t_{d}\right) \in \mathbb{R}^{d}$ and $\langle\cdot, \cdot\rangle$ denotes the scalar product in $\mathbb{R}^{d}$.

Since $\left(X_{i}\right)_{1 \leqslant i \leqslant d}$ has Poisson marginals, all marginals of $\mu$ on $\mathbb{R}^{d}$ are supported by $\{0,1\}$ and consequently the Lévy measure $\mu(d x)$ is supported on the vertices of the unit hypercube of $\mathbb{R}^{d}$ and takes the form

$$
\mu(d x)=\sum_{\emptyset \neq S \subset\{1,2, \ldots, d\}} a_{S} \delta_{e_{S}}(d x),
$$

where $\left(a_{S}\right)_{\emptyset \neq S \subset\{1,2, \ldots, d\}}$ is a family of nonnegative numbers and

$$
C_{d}=\{0,1\}^{d}=\left\{e_{S}:=\sum_{i \in S} e_{i}: S \subset\{1, \ldots, d\}\right\}
$$

denotes the vertices of the $d$-dimensional unit hypercube, identified to the power set $\{0,1\}^{d} \simeq\{S \in\{1, \ldots, d\}\}$ of $\{1, \ldots, d\}$, and $\left(e_{k}\right)_{1 \leqslant k \leqslant d}$ is the canonical basis of $\mathbb{R}^{d}$.

Consequently, any $d$-dimensional Poisson random vector $X=\left(X_{1}, \ldots, X_{d}\right)$ can be represented as

$$
X_{i}=\sum_{\substack{S \in\{0,1\} d \\ S \neq 0}} \mathbf{1}_{\{i \in S\}} X_{S}=\sum_{\substack{S \subset\{1,2, \ldots, d\} \\ S \ni i}} X_{S}, \quad i=1, \ldots, d,
$$

where $\left(X_{S}\right)_{\emptyset \neq S \subset\{1,2, \ldots, d\}}$ is a family of $2^{d}-1$ independent Poisson random variables with respective intensities $\left(a_{S}\right)_{\emptyset \neq S \subset\{1,2, \ldots, d\}}$, cf. also Theorem 3 of [5].

In particular, at most $2^{d}-1$ degrees of freedom are needed in order to characterize the probability distribution of $X$. Note also that

$$
\operatorname{Cov}\left(X_{i}, X_{j}\right)=\sum_{\substack{S \subset\{1,2, \ldots, d\} \\ S \ni i, j}} \operatorname{Var}\left[X_{S}\right] \geqslant 0, \quad i, j=1, \ldots, d,
$$


cf. also Theorem 4 of [5], showing that $X$ has positive covariances.

For example when $d=2$ we have

$$
\left\{\begin{array}{l}
X_{1}=X_{\{1\}}+X_{\{1,2\}} \\
X_{2}=X_{\{2\}}+X_{\{1,2\}}
\end{array}\right.
$$

and when $d=3$ we find

$$
\left\{\begin{array}{lllll}
X_{1}=X_{\{1\}} & +X_{\{1,2\}} & +X_{\{1,3\}} & & +X_{\{1,2,3\}} \\
X_{2}=X_{\{2\}} & +X_{\{1,2\}} & & +X_{\{2,3\}} & +X_{\{1,2,3\}} \\
X_{3}=X_{\{3\}} & & +X_{\{1,3\}} & +X_{\{2,3\}} & +X_{\{1,2,3\}} .
\end{array}\right.
$$

By decomposing the sum (2.1) according to the $\left(\begin{array}{l}d \\ i\end{array}\right)$ subsets $S_{i}^{1}, \ldots, S_{i}^{\left(\begin{array}{c}d \\ i\end{array}\right)}$ of $\{1, \ldots, d\}$ of sizes $i=1, \ldots, d$ we can also write

$$
X_{i}=\sum_{i=1}^{d} \sum_{j=1}^{\left(\begin{array}{c}
d \\
i
\end{array}\right)} \mathbf{1}_{\left\{i \in S_{i}^{j}\right\}} X_{S_{i}^{j}}, \quad i=1, \ldots, d .
$$

\section{Triangular Poisson arrays}

Since Poisson random vectors can depend on $2^{d}-1$ degrees of freedom $\left(a_{S}\right)_{\emptyset \neq S \subset\{1,2, \ldots, d\}}$, it should be generally not possible to characterize their ordering based on the data of its covariance matrix which contains only $d(d+1) / 2$ components.

In this paper we restrict ourselves to $d$-dimensional Poisson random vectors $\left(X_{i}\right)_{1 \leqslant i \leqslant d}$ whose Lévy measure $\mu(d x)$ is supported by

$$
\left\{\mathbf{e}_{i, j}:=e_{1}+\cdots+e_{i-1}+e_{j}: 1 \leqslant i \leqslant j \leqslant d\right\}
$$

i.e. $\mu(d x)$ takes the form

$$
\mu(d x)=\sum_{1 \leqslant i \leqslant j \leqslant d} a_{i, j} \delta_{\mathbf{e}_{i, j}}(d x)
$$

where

$$
\mathbf{e}_{i, j}=(1, \ldots, \underset{\substack{\uparrow \\ i-1}}{1}, 0, \ldots, 0, \underset{\substack{\uparrow \\ j}}{1}, 0, \ldots, 0), \quad 1 \leqslant i \leqslant j \leqslant d
$$


and the vector $\left(X_{i}\right)_{1 \leqslant i \leqslant d}$ actually depends on $d(d+1) / 2$ parameters $\left(a_{i, j}\right)_{1 \leqslant i \leqslant j \leqslant d}$.

In other words the $d$-dimensional Poisson random vector $\left(X_{i}\right)_{1 \leqslant i \leqslant d}$ with Lévy measure $\mu$ given by (3.1) can be represented as

$$
X=\sum_{1 \leqslant k \leqslant l \leqslant d} X_{k, l} \mathbf{e}_{k, l},
$$

where $\left(X_{i, j}\right)_{1 \leqslant i \leqslant j \leqslant d}$ is a triangular array of independent Poisson random variables with respective parameters $\left(a_{i, j}\right)_{1 \leqslant i \leqslant j \leqslant d}$, and we have

$$
X_{i}=\sum_{k=1}^{i} X_{k, i}+\sum_{k=i+1}^{d} \sum_{l=k}^{d} X_{k, l}
$$

$i=1, \ldots, d$.

For example when $d=2$ we have

$$
\left\{\begin{array}{l}
X_{1}=X_{1,1}+X_{2,2} \\
X_{2}=X_{1,2}+X_{2,2}
\end{array}\right.
$$

which coincides with (2.2), and when $d=3$ we get

$$
\left\{\begin{array}{lll}
X_{1}=X_{1,1}+X_{2,2} & +X_{2,3} & +X_{3,3} \\
X_{2}=X_{1,2}+X_{2,2} & & +X_{3,3} \\
X_{3}=X_{1,3} & +X_{2,3} & +X_{3,3}
\end{array}\right.
$$

which, in comparison with (2.3), excludes an interaction component specific to rows 2 and 3. Note that the 2-dimensional case (3.4) does not impose any such restriction on the form of the vector $X$.

From (3.3) we have

$$
\operatorname{Cov}\left(X_{i}, X_{i}\right)=E\left[X_{i}\right]=\sum_{k=1}^{i} a_{k, i}+\sum_{k=i+1}^{d} \sum_{l=k}^{d} a_{k, l},
$$

and

$$
\operatorname{Cov}\left(X_{i}, X_{j}\right)=\sum_{k=i+1}^{j} a_{k, j}+\sum_{k=j+1}^{d} \sum_{l=k}^{d} a_{k, l}, \quad 1 \leqslant i<j \leqslant d,
$$


hence the vector $C=\left(\operatorname{Cov}\left(X_{i}, X_{j}\right)\right)_{1 \leqslant i \leqslant j \leqslant d}$ is obtained from $A=\left(a_{k, l}\right)_{1 \leqslant k \leqslant l \leqslant d}$ and the matrix transformation $C=M \times A$, i.e.

$$
\operatorname{Cov}\left(X_{i}, X_{j}\right)=\sum_{1 \leqslant j \leqslant k \leqslant d} a_{k, l} M_{i, j, k, l}, \quad 1 \leqslant i \leqslant j \leqslant d,
$$

where the $(d(d+1) / 2)^{2}$-dimensional matrix $M=\left(M_{i, j, k, l}\right)_{\substack{1 \leqslant i \leqslant j \leqslant d \\ 1 \leqslant k \leqslant d}}$ is given by

$$
M_{i, j, k, l}=\mathbf{1}_{\{j+1 \leqslant k \leqslant l \leqslant d\}}+\mathbf{1}_{\{1 \leqslant k \leqslant i, i=j=l\}}+\mathbf{1}_{\{i+1 \leqslant k \leqslant j, i<j=l\}} .
$$

We can check by an elementary computation that the inverse $M^{-1}$ of $M$ is given by the inversion formula

$$
a_{k, l}= \begin{cases}\operatorname{Cov}\left(X_{1}, X_{1}\right)+\sum_{j=3}^{d} \operatorname{Cov}\left(X_{2}, X_{j}\right)-\sum_{j=2}^{d} \operatorname{Cov}\left(X_{1}, X_{j}\right), & k=l=1, \\ \operatorname{Cov}\left(X_{l}, X_{l}\right)-\operatorname{Cov}\left(X_{1}, X_{l}\right), & 2 \leqslant k<l \leqslant d, 2 \leqslant l \leqslant d, \\ \operatorname{Cov}\left(X_{k-1}, X_{l}\right)-\operatorname{Cov}\left(X_{k}, X_{l}\right), & \\ \operatorname{Cov}\left(X_{k-1}, X_{k}\right)+\sum_{j=k+2}^{d} \operatorname{Cov}\left(X_{k+1}, X_{j}\right)-\sum_{j=k+1}^{d} \operatorname{Cov}\left(X_{k}, X_{j}\right), & 2 \leqslant k=l \leqslant d,\end{cases}
$$

with, for $d=2$,

$$
\left\{\begin{array}{l}
a_{1,1}=\operatorname{Cov}\left(X_{1}, X_{1}\right)-\operatorname{Cov}\left(X_{1}, X_{2}\right), \\
a_{1,2}=\operatorname{Cov}\left(X_{2}, X_{2}\right)-\operatorname{Cov}\left(X_{1}, X_{2}\right), \\
a_{2,2}=\operatorname{Cov}\left(X_{1}, X_{2}\right) .
\end{array}\right.
$$

In particular it follows from (3.7) that the distribution of $X$ given by (3.3) is characterized by its covariance matrix $\left(\operatorname{Cov}\left(X_{i}, X_{j}\right)\right)_{1 \leqslant i \leqslant j \leqslant d}$, and the vector $X$ admits the representation (3.2) provided its covariances yield non-negative values of $\left(a_{k, l}\right)_{1 \leqslant k \leqslant l \leqslant d}$ in (3.7). Such conditions differ from the ones imposed in relation with the triangular representation of [11].

In the simple case $a_{i, j}=0,1 \leqslant i<j \leqslant d$, we have

$$
X_{i}=\sum_{k=i}^{d} X_{k, k}, \quad i=1, \ldots, d,
$$

and $a_{i, i}=\operatorname{Var}\left[X_{i}\right]=\operatorname{Var}\left[X_{i, i}\right]+\cdots+\operatorname{Var}\left[X_{d, d}\right], i=1, \ldots, d$. 


\section{Supermodular Poisson arrays}

When $d=2$ we note that from (3.3)-(3.6) we have

$$
E\left[X_{1}\right]=a_{1,1}+a_{2,2}, \quad E\left[X_{2}\right]=a_{1,2}+a_{2,2}, \quad \text { and } \quad \operatorname{Cov}\left(X_{1}, X_{2}\right)=a_{2,2},
$$

hence the Lévy measure $\mu(d x)$ of any 2-dimensional Poisson random vector $X=$ $\left(X_{1}, X_{2}\right)$ can be represented as

$$
\begin{aligned}
& \int_{\mathbb{R}^{2}} \phi(x) \mu(d x)=\phi(1,0) a_{1,1}+\phi(0,1) a_{1,2}+\phi(1,1) a_{2,2} \\
& \quad=\phi(1,0)\left(E\left[X_{1}\right]-\operatorname{Cov}\left(X_{1}, X_{2}\right)\right)+\phi(0,1)\left(E\left[X_{2}\right]-\operatorname{Cov}\left(X_{1}, X_{2}\right)\right)+\phi(1,1) \operatorname{Cov}\left(X_{1}, X_{2}\right) \\
& \quad=E\left[X_{1}\right] \phi(1,0)+E\left[X_{2}\right] \phi(0,1)+\operatorname{Cov}\left(X_{1}, X_{2}\right)(\phi(1,1)-\phi(1,0)-\phi(0,1)),
\end{aligned}
$$

for any function $\phi: C_{2} \longrightarrow \mathbb{R}$ such that $\phi(0)=0$.

More generally, for all $d \geqslant 2$, the next Lemma 4.1 provides a representation of Lévy measures which will be used in the proof of our main result Theorem 4.2 below.

Lemma 4.1. Assume that $X$ is a Poisson array represented as in (3.2), with Lévy measure $\mu$. Then for any function $\phi: C_{d} \longrightarrow \mathbb{R}$ such that $\phi(0)=0$ we have

$\int_{\mathbb{R}^{d}} \phi(x) \mu(d x)=\sum_{j=1}^{d} E\left[X_{j}\right] \phi\left(\mathbf{e}_{1, j}\right)+\sum_{1 \leqslant i<j \leqslant d} \operatorname{Cov}\left(X_{i}, X_{j}\right)\left(\phi\left(\mathbf{e}_{i+1, j}\right)+\phi\left(\mathbf{e}_{i-1, i-1}\right)-\phi\left(\mathbf{e}_{i, i}\right)-\phi\left(\mathbf{e}_{i, j}\right)\right)$,

with $\mathbf{e}_{0,0}:=(0, \ldots, 0)$.

Proof. We have

$$
\begin{aligned}
\sum_{1 \leqslant i<j \leqslant d} & \operatorname{Cov}\left(X_{i}, X_{j}\right)\left(\phi\left(\mathbf{e}_{i+1, j}\right)+\phi\left(\mathbf{e}_{i-1, i-1}\right)-\phi\left(\mathbf{e}_{i, i}\right)-\phi\left(\mathbf{e}_{i, j}\right)\right) \\
= & \left(\sum_{j=3}^{d} \operatorname{Cov}\left(X_{2}, X_{j}\right)-\sum_{j=2}^{d} \operatorname{Cov}\left(X_{1}, X_{j}\right)\right) \phi\left(\mathbf{e}_{1,1}\right)-\sum_{j=2}^{d} \operatorname{Cov}\left(X_{1}, X_{j}\right) \phi\left(\mathbf{e}_{1, j}\right) \\
& +\sum_{i=2}^{d}\left(\operatorname{Cov}\left(X_{i-1}, X_{i}\right)+\sum_{j=i+2}^{d} \operatorname{Cov}\left(X_{i+1}, X_{j}\right)-\sum_{j=i+1}^{d} \operatorname{Cov}\left(X_{i}, X_{j}\right)\right) \phi\left(\mathbf{e}_{i, i}\right) \\
& +\sum_{2 \leqslant i<j \leqslant d}\left(\operatorname{Cov}\left(X_{i-1}, X_{j}\right)-\operatorname{Cov}\left(X_{i}, X_{j}\right)\right) \phi\left(\mathbf{e}_{i, j}\right)
\end{aligned}
$$




$$
=-\sum_{j=1}^{d} E\left[X_{j}\right] \phi\left(\mathbf{e}_{1, j}\right)+\sum_{1 \leqslant i \leqslant j \leqslant d} a_{i, j} \phi\left(\mathbf{e}_{i, j}\right),
$$

where the conclusion comes from the above inversion formula (3.7).

The above Lemma 4.1 shows that the transpose $M^{-1 \dagger}$ of $M^{-1}$ is given by

$$
M_{i, j, k, l}^{-1 \dagger}=\mathbf{1}_{\{k=1 \leqslant i=j=l\}}+\mathbf{1}_{\{k=i+1 \leqslant l=j\}}+\mathbf{1}_{\{k=l=i-1<j-1\}}-\mathbf{1}_{\{k=l=i<j\}}-\mathbf{1}_{\{k=i<l=j\}},
$$

and we have

$$
\begin{aligned}
\int_{\mathbb{R}^{d}} \phi(x) \mu(d x) & =\sum_{1 \leqslant k \leqslant l \leqslant d} a_{k, l} \phi\left(\mathbf{e}_{k, l}\right) \\
& =\sum_{1 \leqslant k \leqslant l \leqslant d} \phi\left(\mathbf{e}_{k, l}\right) \sum_{1 \leqslant i \leqslant j \leqslant d} M_{k, l, i, j}^{-1} \operatorname{Cov}\left(X_{i}, X_{j}\right) \\
& =\sum_{1 \leqslant i \leqslant j \leqslant d} \operatorname{Cov}\left(X_{i}, X_{j}\right) \sum_{1 \leqslant k \leqslant l \leqslant d} M_{k, l, i, j}^{-1} \phi\left(\mathbf{e}_{k, l}\right) \\
& =\sum_{1 \leqslant i \leqslant j \leqslant d} \operatorname{Cov}\left(X_{i}, X_{j}\right) \sum_{1 \leqslant k \leqslant l \leqslant d} M_{i, j, k, l}^{-1 \dagger} \phi\left(\mathbf{e}_{k, l}\right) .
\end{aligned}
$$

Consider now two Poisson arrays $X$ and $Y$ whose respective Lévy measures $\mu$ and $\nu$ are represented as

$$
\mu(d x)=\sum_{1 \leqslant i \leqslant j \leqslant d} a_{i, j} \delta_{\mathbf{e}_{i, j}}(d x) \text { and } \nu(d x)=\sum_{1 \leqslant i \leqslant j \leqslant d} b_{i, j} \delta_{\mathbf{e}_{i, j}}(d x),
$$

as in (3.1). If $X_{i}$ has the same distribution as $Y_{i}$ for all $i=1, \ldots, d$ then $E\left[X_{i}\right]=E\left[Y_{i}\right]$, $i=1, \ldots, d$, and Lemma 4.1 shows that

$$
\begin{aligned}
\int_{\mathbb{R}^{d}} \phi(x) \nu(d x)-\int_{\mathbb{R}^{d}} \phi(x) \mu(d x) & \\
= & \sum_{1 \leqslant i<j \leqslant d}\left(\operatorname{Cov}\left(Y_{i}, Y_{j}\right)-\operatorname{Cov}\left(X_{i}, X_{j}\right)\right)\left(\phi\left(\mathbf{e}_{i+1, j}\right)+\phi\left(\mathbf{e}_{i-1, i-1}\right)-\phi\left(\mathbf{e}_{i, i}\right)-\phi\left(\mathbf{e}_{i, j}\right)\right) \\
= & \sum_{1 \leqslant i<j \leqslant d}\left(\operatorname{Cov}\left(Y_{i}, Y_{j}\right)-\operatorname{Cov}\left(X_{i}, X_{j}\right)\right) t_{i, j} \phi\left(\mathbf{e}_{i-1, i-1}\right),
\end{aligned}
$$

where $t_{i, j}$ is the linear mapping defined on $\phi$ by

$$
t_{i, j} \phi(z)=\phi\left(z+e_{i}+e_{j}\right)+\phi(z)-\phi\left(z+e_{i}\right)-\phi\left(z+e_{j}\right)
$$

and

$t_{i, j} \phi\left(\mathbf{e}_{i-1, i-1}\right)=\phi\left(\mathbf{e}_{i-1, i-1}+e_{i}+e_{j}\right)+\phi\left(\mathbf{e}_{i-1, i-1}\right)-\phi\left(\mathbf{e}_{i-1, i-1}+e_{i}\right)-\phi\left(\mathbf{e}_{i-1, i-1}+e_{j}\right)$ 


$$
=\phi\left(\mathbf{e}_{i+1, j}\right)+\phi\left(\mathbf{e}_{i-1, i-1}\right)-\phi\left(\mathbf{e}_{i, i}\right)-\phi\left(\mathbf{e}_{i, j}\right) .
$$

In other words, (4.1) provides the explicit form of the decomposition stated in Theorem 1 of $[6]$.

Relation (4.1) also shows that the nonnegativity of the coefficients

$$
\operatorname{Cov}\left(Y_{i}, Y_{j}\right)-\operatorname{Cov}\left(X_{i}, X_{j}\right) \geqslant 0, \quad 1 \leqslant i<j \leqslant d
$$

becomes a necessary and sufficient condition for the supermodular ordering of the Lévy measures $\mu$ and $\nu$ which here have finite support.

Next is the main result of this paper. It reformulates (4.2) as a necessary and sufficient condition for supermodular ordering of triangular Poisson arrays, based on Theorem 4.6 of [2] which allows us to carry over the notion of supermodularity from the finite support setting of Lévy measures $\mu, \nu$ on the cube $C_{d}$, to the infinite support setting of Poisson random variables.

Theorem 4.2. Consider two Poisson arrays $X$ and $Y$ both represented as in (3.2). Then the conditions

$$
E\left[X_{i}\right]=E\left[Y_{i}\right], \quad 1 \leqslant i \leqslant d
$$

and

$$
\operatorname{Cov}\left(X_{i}, X_{j}\right) \leqslant \operatorname{Cov}\left(Y_{i}, Y_{j}\right), \quad 1 \leqslant i<j \leqslant d,
$$

are necessary and sufficient for the supermodular ordering $X \leqslant_{\mathrm{sm}} Y$.

Proof. By Theorem 4.6 in [2] it suffices to show that

$$
\int_{\mathbb{R}^{d}} \phi(x) \mu(d x) \leqslant \int_{\mathbb{R}^{d}} \phi(y) \nu(d y)
$$

for all supermodular functions $\phi: \mathbb{R}^{d} \longrightarrow \mathbb{R}$ satisfying $\phi(0)=\phi\left(\mathbf{e}_{0,0}\right)=0$, where $\mu(d x)$ and $\nu(d y)$ respectively denote the Lévy measures of $X$ and $Y$. By Lemma 4.1 we have the identity

$$
\int_{\mathbb{R}^{d}} \phi(x) \nu(d x)-\int_{\mathbb{R}^{d}} \phi(x) \mu(d x)
$$




$$
=\sum_{1 \leqslant i<j \leqslant d}\left(\operatorname{Cov}\left(Y_{i}, Y_{j}\right)-\operatorname{Cov}\left(X_{i}, X_{j}\right)\right)\left(\phi\left(\mathbf{e}_{i+1, j}\right)+\phi\left(\mathbf{e}_{i-1, i-1}\right)-\phi\left(\mathbf{e}_{i, i}\right)-\phi\left(\mathbf{e}_{i, j}\right)\right)
$$

Next, the supermodular inequalities

$$
\phi\left(\mathbf{e}_{i, i}\right)+\phi\left(\mathbf{e}_{i, j}\right) \leqslant \phi\left(\mathbf{e}_{i, i} \wedge \mathbf{e}_{i, j}\right)+\phi\left(\mathbf{e}_{i, i} \vee \mathbf{e}_{i, j}\right)=\phi\left(\mathbf{e}_{i-1, i-1}\right)+\phi\left(\mathbf{e}_{i+1, j}\right)
$$

show that

$\left(\operatorname{Cov}\left(Y_{i}, Y_{j}\right)-\operatorname{Cov}\left(X_{i}, X_{j}\right)\right)\left(\phi\left(\mathbf{e}_{i+1, j}\right)+\phi\left(\mathbf{e}_{i-1, i-1}\right)-\phi\left(\mathbf{e}_{i, i}\right)-\phi\left(\mathbf{e}_{i, j}\right)\right) \geqslant 0, \quad 1 \leqslant i<j \leqslant d$, under condition (4.4), which allows us to conclude by (4.5).

Note that in view of Condition (4.3), the inequality (4.4) can be rewritten using joint moments, as

$$
E\left[X_{i} X_{j}\right] \leqslant E\left[Y_{i} Y_{j}\right], \quad 1 \leqslant i<j \leqslant d
$$

The next proposition is relative to the increasing supermodular order.

Proposition 4.3. Consider two Poisson arrays $X$ and $Y$ both represented as in (3.2), and assume that

$$
E\left[X_{i}\right] \leqslant E\left[Y_{i}\right], \quad 1 \leqslant i \leqslant d
$$

and

$$
\operatorname{Cov}\left(X_{i}, X_{j}\right) \leqslant \operatorname{Cov}\left(Y_{i}, Y_{j}\right), \quad 1 \leqslant i<j \leqslant d
$$

Then we have

$$
E[\Phi(X)] \leqslant E[\Phi(Y)]
$$

for all nondecreasing supermodular functions $\Phi: \mathbb{R}^{d} \longrightarrow \mathbb{R}$.

Proof. First we note that Theorem 4.6 of [2] can be easily extended to nondecreasing supermodular functions $\phi$ on $\mathbb{R}^{d}$ satisfying $\phi(0)=0$, using the same approximation as in Lemma 4.3 therein. The conclusion follows again from Lemma 4.1 and the decomposition

$$
\begin{gathered}
\int_{\mathbb{R}^{d}} \phi(x) \nu(d x)-\int_{\mathbb{R}^{d}} \phi(x) \mu(d x) \\
=\sum_{j=1}^{d}\left(E\left[Y_{j}\right]-E\left[X_{j}\right]\right) \phi\left(\mathbf{e}_{1, j}\right)
\end{gathered}
$$




$$
+\sum_{1 \leqslant i<j \leqslant d}\left(\operatorname{Cov}\left(Y_{i}, Y_{j}\right)-\operatorname{Cov}\left(X_{i}, X_{j}\right)\right)\left(\phi\left(\mathbf{e}_{i+1, j}\right)+\phi\left(\mathbf{e}_{i-1, i-1}\right)-\phi\left(\mathbf{e}_{i, i}\right)-\phi\left(\mathbf{e}_{i, j}\right)\right),
$$

for all nondecreasing supermodular functions $\phi: C_{d} \longrightarrow \mathbb{R}$, since $\phi\left(\mathbf{e}_{1, j}\right) \geqslant \phi(0)=0$, $j=1, \ldots, d$.

\section{Sums of Gaussian and Poisson vectors}

Theorem 4.2 can be extended to a sufficient condition for the sum of Gaussian and Poisson vectors, as follows.

Theorem 4.4. Consider two Poisson arrays $X$ and $Y$ both represented as in (3.2), and two d-dimensional centered Gaussian random vectors $U, V$ such that $U$ is independent of $X$ and $V$ is independent of $Y$. Then the conditions $E\left[X_{i}\right]=E\left[Y_{i}\right]$, $1 \leqslant i \leqslant d$, and

$$
\operatorname{Cov}\left(U_{i}, U_{j}\right) \leqslant \operatorname{Cov}\left(V_{i}, V_{j}\right), \quad \operatorname{Cov}\left(X_{i}, X_{j}\right) \leqslant \operatorname{Cov}\left(Y_{i}, Y_{j}\right)
$$

$1 \leqslant i<j \leqslant d$, are sufficient for the supermodular ordering $X+U \leqslant \mathrm{sm} Y+V$.

Proof. This is a consequence of Theorem 4.2 of [9] on Gaussian random vectors, Theorem 4.2 above, and the fact that the supermodular ordering is closed under convolution, cf. Theorem 3.9.14-(C) of [10].

Proposition 4.3 can be similarly extended to sums of Gaussian vectors and Poisson arrays.

As an application of Theorem 4.4 we can consider exponential jump-diffusion models $\left(X_{t}^{1}, \ldots, X_{t}^{d}\right)_{t \in \mathbb{R}_{+}}$of the form

$$
X_{t}^{i}=X_{0}^{i} \exp \left(\sigma_{i} B_{t}^{i}+\eta_{i} Z_{t}^{i}+\mu_{i} t\right), \quad i=1, \ldots, d,
$$

as in (3.3), where $Z_{t}^{i}$ is the Poisson jump process

$$
Z_{t}^{i}=\sum_{k=1}^{i} N_{t}^{k, i}+\sum_{k=i+1}^{d} \sum_{l=k}^{d} N_{t}^{k, l}, \quad i=1, \ldots, d,
$$

and $\left\{\left(N_{t}^{k, l}\right)_{t \in \mathbb{R}_{+}}, 1 \leqslant k \leqslant l \leqslant d\right\}$ is a family of $d(d+1) / 2$ independent standard Poisson processes with respective intensities $\left(a_{k, l}\right)_{1 \leqslant k \leqslant l \leqslant d},\left(B_{t}^{1}, \ldots, B_{t}^{d}\right)_{t \in \mathbb{R}_{+}}$is a $d$-dimensional 
Browian motion, $X_{0}^{i}, \sigma_{i}, \eta_{i}$ are nonnegative coefficients, and $\mu_{i} \in \mathbb{R}, i=1, \ldots, d$. In that case, Theorem 4.4 shows that the supermodular ordering of vectors of the form $\left(X_{t}^{1}, \ldots, X_{t}^{d}\right)$ follows from the componentwise orderings of the covariance matrices of the $d$-dimensional Brownian and Poisson processes $\left(B_{t}^{1}, \ldots, B_{t}^{d}\right)$ and $\left(Z_{t}^{1}, \ldots, Z_{t}^{d}\right)$, cf. Theorem 3.9.8-(P8) of [10], since every exponential function in (4.7) is increasing.

\section{Convex ordering}

We end this paper by some comments in the case of the convex ordering.

Proposition 4.5. Consider two Poisson arrays $X$ and $Y$ both represented as in (3.2). Then we have $X \leqslant_{\mathrm{cx}} Y$ if and only if $X$ and $Y$ have same distributions.

Proof. Assume $X \leqslant_{\mathrm{cx}} Y$, i.e. we have

$$
E[\Phi(X)] \leqslant E[\Phi(Y)]
$$

for all convex functions $\Phi: \mathbb{R}^{d} \longrightarrow \mathbb{R}$. Clearly this implies $E\left[X_{k}\right]=E\left[Y_{k}\right], k=$ $1, \ldots, d$. Next, letting $0 \leqslant k<l \leqslant d$ and assuming that $\operatorname{Cov}\left(Y_{i}, Y_{j}\right)-\operatorname{Cov}\left(X_{i}, X_{j}\right)=0$ whenever $1 \leqslant i \leqslant k \leqslant d$ and $1 \leqslant i<j \leqslant l \leqslant d$, we check that the function

$$
\left(x_{1}, \ldots, x_{d}\right) \mapsto \phi_{k, l+1}\left(x_{1}, \ldots, x_{d}\right):=\max \left(0,2 x_{l+1}-\sum_{a=k}^{d} x_{a}\right)
$$

is convex on $\mathbb{R}^{d}$. In addition, taking again $\mathbf{e}_{i, j}:=e_{1}+\cdots+e_{i-1}+e_{j}, 1 \leqslant i \leqslant j \leqslant d$, we note that it satisfies $\phi_{k, l+1}\left(\mathbf{e}_{i, j}\right)=1$ if and only if $1 \leqslant i \leqslant k<l+1=j \leqslant d$, otherwise $\phi_{k, l+1}\left(\mathbf{e}_{i, j}\right)=0$, hence

$$
\phi_{k, l+1}\left(\mathbf{e}_{k+1, l+1}\right)+\phi_{k, l+1}\left(\mathbf{e}_{k-1, k-1}\right)-\phi_{k, l+1}\left(\mathbf{e}_{k, k}\right)-\phi_{k, l+1}\left(\mathbf{e}_{k, l+1}\right)<0,
$$

while

$$
\phi_{k, l+1}\left(\mathbf{e}_{i+1, j}\right)+\phi_{k, l+1}\left(\mathbf{e}_{i-1, i-1}\right)-\phi_{k, l+1}\left(\mathbf{e}_{i, i}\right)-\phi_{k, l+1}\left(\mathbf{e}_{i, j}\right)=0
$$

whenever $1 \leqslant k<i \leqslant d$ or $2 \leqslant l+1<j \leqslant d$. Hence from Lemma 4.1, the condition $\operatorname{Cov}\left(Y_{k}, Y_{l+1}\right)>\operatorname{Cov}\left(X_{k}, X_{l+1}\right)$ implies

$\int_{\mathbb{R}^{d}} \phi(x) \nu(d x)-\int_{\mathbb{R}^{d}} \phi(x) \mu(d x)$ 


$$
\begin{aligned}
& =\sum_{1 \leqslant i<j \leqslant d}\left(\operatorname{Cov}\left(Y_{i}, Y_{j}\right)-\operatorname{Cov}\left(X_{i}, X_{j}\right)\right)\left(\phi\left(\mathbf{e}_{i+1, j}\right)+\phi\left(\mathbf{e}_{i-1, i-1}\right)-\phi\left(\mathbf{e}_{i, i}\right)-\phi\left(\mathbf{e}_{i, j}\right)\right) \\
& =\left(\operatorname{Cov}\left(Y_{k}, Y_{l+1}\right)-\operatorname{Cov}\left(X_{k}, X_{l+1}\right)\right)\left(\phi\left(\mathbf{e}_{k+1, l+1}\right)+\phi\left(\mathbf{e}_{k-1, k-1}\right)-\phi\left(\mathbf{e}_{k, k}\right)-\phi\left(\mathbf{e}_{k, l+1}\right)\right) \\
& <0,
\end{aligned}
$$

which is a contradiction. After proceeding similarly with $\phi_{k+1, l}$, we conclude by induction that $\operatorname{Cov}\left(Y_{k}, Y_{l}\right)-\operatorname{Cov}\left(X_{k}, X_{l}\right)=0$ for all $1 \leqslant k<l \leqslant d$.

\section{References}

[1] M. Arnaudon, J.-C. Breton, and N. Privault. Convex ordering for random vectors using predictable representation. Potential Anal., 29(4):327-349, 2008.

[2] N. Bäuerle, A. Müller, and A. Blatter. Dependence properties and comparison results for Lévy processes. Math. Meth. Operat. Res., 67:161-186, 2008.

[3] J. Bergenthum and L. Rüschendorf. Comparison of semimartingales and Lévy processes. Ann. Probab., 35(1), 2007.

[4] C. Houdré, V. Pérez-Abreu, and D. Surgailis. Interpolation, correlation identities, and inequalities for infinitely divisible variables. J. Fourier Anal. Appl., 4(6):651-668, 1998.

[5] K. Kawamura. The structure of multivariate Poisson distribution. Kodai Math. J., 2(3):337-345, 1979.

[6] M.A Meyer and B. Strulovici. The supermodular stochastic ordering. CEPR Discussion Paper DP9486, May 2013. http://www.cepr.org/pubs/dps/DP9486.

[7] A. Müller. Stop-loss order for portfolios of dependent risks. Insurance Math. Econom., 21(3):219-223, 1997.

[8] A. Müller. Stochastic ordering of multivariate normal distributions. Ann. Inst. Statist. Math., $53(3): 567-575,2001$.

[9] A. Müller and M. Scarsini. Some remarks on the supermodular order. J. Multivariate Anal., 73:107-119, 2000.

[10] A. Müller and D. Stoyan. Comparison methods for stochastic models and risks. Wiley Series in Probability and Statistics. John Wiley \& Sons, Ltd., Chichester, 2002.

[11] C.H. Sim. Generation of Poisson and gamma random vectors with given marginals and covariance matrix. Journal of Statistical Computation and Simulation, 47:1-10, 1993.

[12] H. Teicher. On the multivariate Poisson distribution. Skand. Aktuarietidskr., 37:1-9, 1954. 